\title{
Numerical Solution to Maxwell's Equations in Singular Waveguides
}

\author{
Franck Assous ${ }^{1}$ and Patrick Ciarlet Jr. ${ }^{2}$ \\ ${ }^{1}$ Bar-Ilan University, 52900 Ramat-Gan, Israel \\ and College of Judea\&Samaria, Ariel, Israel \\ franckassous@netscape.net \\ ${ }^{2}$ ENSTA,32 bvd Victor, 75739, Paris Cedex 15 \\ ciarlet@ensta.fr
}

\begin{abstract}
This paper is devoted to the numerical solution of the instationary Maxwell equations in singular waveguides. The geometry is called singular, as its boundary includes reentrant corners or edges, which generate, in their neighborhood, strong electromagnetic fields. We have built a method which allows to compute the time-dependent electromagnetic field, based on a splitting of the spaces of solutions: First, the subspace of regular fields, which coincides with the whole space of solutions, in the case of convex or smooth boundary; Second, a singular subspace, defined and characterized via the singularities of the Laplace operator. Numerical results illustrate the influence of frequency of the ingoing electromagnetic waves in a L-shaped waveguide.
\end{abstract}

\section{Introduction}

Many practical problems require the computation of electromagnetic fields. They are usually based on Maxwell equations. Within this framework, we developed a numerical method for solving the instationary Maxwell equations (see [5]), with continuous approximations of the electromagnetic field. However, in practical examples, the boundary of the computational domain includes reentrant corners and/or edges, called geometrical singularities because they generate strong fields.

We developed a method, the so-called Singular Complement Method, which consists in splitting the space of electromagnetic fields into a two-term, direct, possibly orthogonal sum. The first subspace is made of regular fields, the second one is called the subspace of singular electromagnetic fields. One compute the regular part of the solution with the help of an ad hoc-classical - method [5]. The singular part is computed with the help of specifically designed methods.

The present paper is a continuation of the Singular Complement Method, developed for Maxwell equations in 2D 4, and for the Vlasov-Maxwell equations 1]. We first recall Maxwell's equations, together with the functional framework, which is then used to describe the Singular Complement Method. Section 3 is devoted to the numerical algorithms. In particular, the computation of singular basis functions is described, together with the discretization of the variational formulations. Numerical experiments are presented in the last Section. 


\section{Mathematical Analysis of the Problem}

Let $\Omega$ be a bounded, open, polyhedral subset of $\mathbb{R}^{3}$. We denote by $\Gamma$ its boundary and by $\mathbf{n}$ the unit outward normal to $\Gamma$. If we let $c, \varepsilon_{0}$ and $\mu_{0}$ be respectively the light velocity, the dielectric permittivity and the magnetic permeability $\left(\varepsilon_{0} \mu_{0} c^{2}=1\right)$, Maxwell's equations in vacuum read,

$$
\begin{aligned}
\frac{\partial \mathcal{E}}{\partial t}-c^{2} \operatorname{curl} \mathcal{B} & =-\frac{1}{\varepsilon_{0}} \mathcal{J}, & \operatorname{div} \mathcal{E} & =\frac{\rho}{\varepsilon_{0}}, \\
\frac{\partial \mathcal{B}}{\partial t}+\operatorname{curl} \mathcal{E} & =0, & \operatorname{div} \mathcal{B} & =0,
\end{aligned}
$$

where $\mathcal{E}$ and $\mathcal{B}$ are the electric and magnetic fields, $\rho$ and $\mathcal{J}$ the charge and current densities, which depend on the space variable $\mathbf{x}$ and on the time variable $t$. As it is well known, $\rho$ and $\mathcal{J}$ have to verify the charge conservation

$$
\frac{\partial \rho}{\partial t}+\operatorname{div} \mathcal{J}=0
$$

These equations are supplemented with appropriate boundary conditions. For the sake of simplicity, we will only consider first perfectly conducting boundary. The case of Silver-Müller boundary condition will be then introduced. For the time being, we suppose that

$$
\mathcal{E} \times \mathbf{n}=0 \text { and } \mathcal{B} \cdot \mathbf{n}=0 \text { on } \Gamma
$$

Finally, one adds initial conditions, set at time $t=0, \mathcal{E}(0)=\mathcal{E}_{0}, \mathcal{B}(0)=\mathcal{B}_{0}$. We explain below how in our formulation the electric and the magnetic fields can be handled separately. Even if the principles of analysis are the same, the results and the mathematical tools are different (see theoretical details in 2]). The electric case generally appears in a non divergence-free modelling, typically the Vlasov-Maxwell equations, and has been exposed in 11. In this paper, we will focus on the magnetic field formulation. Let us recall the definitions of the following spaces

$$
\begin{aligned}
\mathbf{H}(\operatorname{curl}, \Omega) & =\left\{\mathbf{u} \in \mathbf{L}^{2}(\Omega), \operatorname{curl} \mathbf{u} \in \mathbf{L}^{2}(\Omega)\right\}, \\
\mathbf{H}(\operatorname{div}, \Omega) & =\left\{\mathbf{u} \in \mathbf{L}^{2}(\Omega), \operatorname{div} \mathbf{u} \in L^{2}(\Omega)\right\}, \\
\mathbf{H}^{1}(\Omega) & =\left\{\mathbf{u} \in \mathbf{L}^{2}(\Omega), \operatorname{grad} \mathbf{u} \in \mathbf{L}^{2}(\Omega)\right\} .
\end{aligned}
$$

We define the space of magnetic fields $\mathcal{B}$, called $\mathbf{Y}$,

$$
\mathbf{Y}=\left\{\mathbf{y} \in \mathbf{H}(\operatorname{curl}, \Omega) \cap \mathbf{H}(\operatorname{div}, \Omega): \mathbf{y} \cdot \mathbf{n}_{\mid \Gamma}=0\right\} .
$$

In what follows, we use the notation $(\cdot, \cdot)_{0}$ for the usual scalar product in $L^{2}(\Omega)$ or $\mathbf{L}^{2}(\Omega)$, and $(\cdot, \cdot)_{\mathbf{Y}}=(\operatorname{curl} \cdot, \operatorname{curl} \cdot)_{0}+(\operatorname{div} \cdot, \operatorname{div} \cdot)_{0}$ for the one in $\mathbf{Y}$.

When the domain is convex (or with a smooth boundary), the space of magnetic fields $\mathbf{Y}$ is included in $\mathbf{H}^{1}(\Omega)$. That is not the case anymore in a singular 
domain (see for instance [10]). One thus introduces the regular subspace for magnetic fields (indexed with $R$ )

$$
\mathbf{Y}_{R}=\mathbf{Y} \cap \mathbf{H}^{1}(\Omega)
$$

which is actually closed in $\mathbf{Y}[9]$. Hence, one can consider its orthogonal subspace (called singular subspace and indexed with ${ }_{S}$ ), and then define a two-part, direct, and orthogonal sum of the space as

$$
\mathbf{Y}=\mathbf{Y}_{R} \stackrel{\perp \mathbf{Y}}{\oplus} \mathbf{Y}_{S}
$$

As a consequence, one can split an element $\mathbf{y}$ into an orthogonal sum of a regular part and of a singular one, namely $\mathbf{y}=\mathbf{y}_{R}+\mathbf{y}_{S}$. We have now to characterize the singular magnetic fields. Following [9], elements $\mathbf{y}_{S} \in \mathbf{Y}_{S}$ satisfy

$$
\begin{gathered}
\Delta \mathbf{y}_{S}=0 \quad \text { in } \Omega, \\
\mathbf{y}_{S} \cdot \mathbf{n}_{\mid \Gamma}=0 .
\end{gathered}
$$

Now, we suppose that a part $\Gamma_{C}$ of the boundary $\Gamma$ behaves as a perfect conductor, namely $\mathcal{B} \cdot \mathbf{n}_{\mid \Gamma}=0$. On the other part $\Gamma_{A}=\Gamma \backslash \Gamma_{C}$, we have to model the electromagnetic interactions between the domain $\Omega$ and the exterior. One has

$$
(\mathcal{E}-c \mathcal{B} \times \mathbf{n}) \times \mathbf{n}=\mathbf{e}^{\star} \times \mathbf{n} \text { on } \Gamma_{A},
$$

where the surface field $\mathbf{e}^{\star}$ is given. These conditions are known as the SilverMüller boundary conditions. Moreover, the artificial boundary $\Gamma_{A}$ is often splitted into $\Gamma_{A}^{i}$ and $\Gamma_{A}^{a}$. On $\Gamma_{A}^{i}$, we model incoming plane waves by a non-vanishing function $\mathbf{e}^{\star}$, whereas we impose on $\Gamma_{A}^{a}$ an absorbing boundary condition by choosing $\mathbf{e}^{\star}=0$. Without loss of generality, one can choose the location of the artificial boundary $\Gamma_{A}$, in such a way that it does not intersect with a geometrical singularity. Moreover, one can also choose a regular shape for $\Gamma_{A}$. Now, one could consider the space of solutions

$$
\mathbf{Y}^{\Gamma_{A}}=\left\{\mathbf{y} \in \mathbf{H}(\mathbf{c u r l}, \Omega) \cap \mathbf{H}(\operatorname{div}, \Omega): \mathbf{y} \cdot \mathbf{n}_{\mid \Gamma_{C}}=0\right\} .
$$

Then introduce the regular subspace $\mathbf{Y}_{R}^{\Gamma_{A}}=\mathbf{Y}^{\Gamma_{A}} \cap \mathbf{H}^{1}(\Omega)$, and construct the ad hoc orthogonal splitting, in which appears a singular space, say $\mathbf{Y}_{S}^{\Gamma_{A}}$. Nevertheless, it is more interesting from a numerical point of view, to consider the (non-orthogonal) splitting

$$
\mathbf{Y}^{\Gamma_{A}}=\mathbf{Y}_{R}^{\Gamma_{A}} \oplus \mathbf{Y}_{S}
$$

First, since the subspace of singular magnetic fields is $\mathbf{Y}_{S}$, as before. Second, modelling incoming plane waves, or imposing an absorbing boundary condition has no impact, as far as the singular subspace is concerned. It will be sufficient, as soon as $\Gamma_{A}$ is not empty, to add in the variational formulation, integral terms on $\Gamma_{A}$ as for a regular domain $\Omega$. 


\section{$3 \quad$ Numerical Algorithms}

The numerical method consists in computing first the basis of the singular subspace. Then we solve the problem by coupling a classical method (to compute the regular part of the solution) to the linear system, which allows to compute the singular part of the solution.

To compute $\mathbf{y}_{S} \in \mathbf{Y}_{S}$, it is convenient to introduce its divergence- and curlfree parts $\mathbf{w}_{S}$ and $\mathbf{m}_{S}$, which verify the following Helmholtz decomposition

$$
\mathbf{y}_{S}=\mathbf{w}_{S}+\mathbf{m}_{S} .
$$

From now on, we assume that the singular subspace $\mathbf{Y}_{S}$ is finite-dimensional. This is actually the case for a two-dimensional domain $\Omega$, where the dimension of the singular subspace is equal to the number of reentrant corners ( cf. 4]). The three-dimensional case can be written formally as below, but some technical mathematical tools are needed (unclassical spaces, weak trace properties, etc.). We refer the reader to 2 for more theoretical details.

We shall need $s_{N}$ and $s_{D}$, the non-vanishing, singular, harmonic functions, with Neumann and Dirichlet homogeneous boundary condition respectively, solutions to

$$
\begin{aligned}
& \Delta s_{N}=0, \quad \Delta s_{D}=0, \quad \text { in } \Omega \\
& \frac{\partial s_{N}}{\partial \boldsymbol{\nu}}=0, \quad s_{D}=0, \quad \text { on } \Gamma \text {. }
\end{aligned}
$$

Remark that $s_{N}$ and $s_{D}$ are not equal to zero since we are looking for a singular solution, namely with a too poor regularity to be a variational solution. One then introduce $\phi_{S}$ and $\psi_{S}$ respectively solution to

$$
-\Delta \phi_{S}=s_{N}, \quad-\Delta \psi_{S}=s_{D} \quad \text { in } \Omega,
$$

still with Neumann and Dirichlet homogeneous boundary condition. Next, the singular basis functions $\mathbf{y}_{S} \in \mathbf{Y}_{S}$ can be obtained (see [3]) with the relation

$$
\mathbf{w}_{S}=\operatorname{curl} \psi_{S}, \quad \mathbf{m}_{S}=\operatorname{grad} \phi_{S},
$$

together with relation (2). Hence, the keypoint is to compute $s_{N}$ and $s_{D}$.

Consider, for simplicity reasons, a domain with one reentrant corner. To compute $s_{N}$ and $s_{D}$, we have chosen to use the Principal Part Method. Let us describe it on $s_{D}$. It consists in splitting $s_{D}$ in a regular part $\tilde{s}_{D}$ (which belongs to $H^{1}(\Omega)$ ) and a known singular part $s_{D}^{P}$

$$
s_{D}=s_{D}^{P}+\tilde{s}_{D} .
$$

It is common knowledge that $s_{D}^{P}=r^{-\alpha} \sin (\alpha \theta)$, where $(r, \theta)$ denote the polar coordinates centered on the reentrant corner of angle $\pi / \alpha$. Above, $s_{D}^{P}$ is singular since it belongs to $L^{2}(\Omega)$ but not to $H^{1}(\Omega)$, and verifies $\Delta s_{D}^{P}=0$. One thus computes with a $P^{1}$ finite element method, $\tilde{s}_{D}$ by solving

$$
\begin{aligned}
& \Delta \tilde{s}_{D}=0 \text { in } \Omega, \\
& \tilde{s}_{D}=-s_{D}^{P} \text { on } \Gamma .
\end{aligned}
$$


Next, one proceeds similarly for the function $\psi_{S} \in H_{0}^{1}(\Omega)$ solution to

$$
\begin{aligned}
-\Delta \psi_{S} & =s_{D} \text { in } \Omega \\
\psi_{S} & =0 \text { on } \Gamma .
\end{aligned}
$$

Again, splitting $\psi_{S}$ in a regular part $\tilde{\psi}_{S}$ (which belongs to $H^{2}(\Omega)$ ) and a singular one $\psi_{S}^{P}$,

$$
\psi_{S}=\tilde{\psi}_{S}+C_{\psi} \psi_{S}^{P}
$$

where $C_{\psi}$ is a constant which can be determined with an integration by parts formula (cf. [3]). One needs the expression of $\psi_{S}^{P}$ in polar coordinates, $\psi_{S}^{P}=$ $r^{\alpha} \sin (\alpha \theta)$. The regular part $\tilde{\psi}_{S}$ is then computed, by solving a standard variational formulation. The singular function $s_{N}$ and $\phi_{S}$ are obtained in the same way.

With the help of singular mappings (see [2]), one can compute the singular electromagnetic basis functions. We get the basis $\mathbf{w}_{S}\left(\right.$ resp. $\mathbf{m}_{S}$ ) by simply taking the curl of $\psi_{S}$ (resp. the gradient of $\phi_{S}$ )

$$
\begin{aligned}
\mathbf{w}_{S} & =\operatorname{curl} \tilde{\psi}_{S}+C_{\psi} \operatorname{curl} \psi_{S}^{P}, \\
\mathbf{m}_{S} & =\nabla \tilde{\phi}_{S}+C_{\phi} \nabla \phi_{S}^{P},
\end{aligned}
$$

and $\mathbf{y}_{S}$ is easily obtained with (2).

We recall now the Variational Formulation, or VF, which have been developed to solve the problem. We also introduce the discretization of this VF. First, Ampère and Faraday's laws are written equivalently as two second-order in time equations, plus suitable initial and boundary conditions. Then, the electric and magnetic fields are decoupled (up to the initial conditions). Next, following [5], we enforce the divergence constraints on the electromagnetic field by introducing two Lagrange multipliers, which dualize Coulomb's and absence of free magnetic monopole's laws. This approach gives a Mixed VF of Maxwell's equations, which is well-posed, if the well-known inf-sup (or Babuska-Brezzi [6]7) condition holds. In addition, we use an Augmented VF, by adding to the bilinear form $(\operatorname{curl} \cdot, \operatorname{curl} \cdot)_{0}$, the term $(\operatorname{div} \cdot \operatorname{div} \cdot)_{0}$. This results in a Mixed, Augmented VF, or $M A V F$. In our case, the magnetic field belongs to $\mathbf{Y}$. Then, the correct Lagrange multiplier space is $L_{0}^{2}(\Omega)$. Denote by $p(t)$ the Lagrange multiplier, this formulation reads

Find $(\mathcal{B}(t), p(t)) \in \mathbf{Y} \times L_{0}^{2}$ such that

$$
\begin{aligned}
& \frac{d^{2}}{d t^{2}}(\mathcal{B}(t), \mathbf{y})_{0}+c^{2}(\mathcal{B}(t), \mathbf{y})_{\mathbf{Y}}+(p(t), \operatorname{div} \mathbf{y})_{0}=\frac{1}{\varepsilon_{0}}(\mathcal{J}(t), \operatorname{curl} \mathbf{y})_{0}, \quad \forall \mathbf{y} \in \mathbf{Y}, \\
& (\operatorname{div} \mathcal{B}(t), q)_{0}=0 \quad \forall q \in L_{0}^{2}(\Omega) .
\end{aligned}
$$


One has to include the regular/singular splitting in this formulation. The magnetic field $\mathcal{B}$ being decomposed into $\mathcal{B}(t)=\mathcal{B}_{R}(t)+\mathcal{B}_{S}(t)$, and the same for the test functions, the variational formulation reads now:

Find $\left(\mathcal{B}_{R}(t), \mathcal{B}_{S}(t), p(t)\right) \in \mathbf{Y}_{R} \times \mathbf{Y}_{S} \times L_{0}^{2}(\Omega)$ such that

$$
\begin{array}{rlrl}
\frac{d^{2}}{d t^{2}}\left(\mathcal{B}_{R}(t), \mathbf{y}_{R}\right)_{0} & +c^{2}\left(\mathcal{B}_{R}(t), \mathbf{y}_{R}\right)_{\mathbf{Y}}+\left(p(t), \operatorname{div} \mathbf{y}_{R}\right)_{0} & =\frac{1}{\varepsilon_{0}}\left(\mathcal{J}(t), \operatorname{curl} \mathbf{y}_{R}\right)_{0} \\
- & -\frac{d^{2}}{d t^{2}}\left(\mathcal{B}_{S}(t), \mathbf{y}_{R}\right)_{0}, & \forall \mathbf{y}_{R} \in \mathbf{Y}_{R} \\
\frac{d^{2}}{d t^{2}}\left(\mathcal{B}_{S}(t), \mathbf{y}_{S}\right)_{0}+c^{2}\left(\mathcal{B}_{S}(t), \mathbf{y}_{S}\right)_{\mathbf{Y}}+\left(p(t), \operatorname{div} \mathbf{y}_{S}\right)_{0}=\frac{1}{\varepsilon_{0}}\left(\mathcal{J}(t), \operatorname{curl} \mathbf{y}_{S}\right)_{0} \\
-\frac{d^{2}}{d t^{2}}\left(\mathcal{B}_{R}(t), \mathbf{y}_{S}\right)_{0}, & \forall \mathbf{y}_{S} \in \mathbf{Y}_{S} \\
\left(\operatorname{div} \mathcal{B}_{R}(t), q\right)_{0}+\left(\operatorname{div} \mathcal{B}_{S}(t), q\right)_{0}=0, & \forall q \in L_{0}^{2}(\Omega) .
\end{array}
$$

Remark 3.1. In the case of a non-orthogonal splitting, as for instance with a non-empty absorbing boundary $\Gamma_{A}$, one has to add $-c^{2}\left(\mathcal{B}_{S}(t), \mathbf{y}_{R}\right)_{\mathbf{Y}}$ to equation (9), and the term $-c^{2}\left(\mathcal{B}_{R}(t), \mathbf{y}_{S}\right)_{\mathbf{Y}}$ to equation (10) (generated by the loss of orthogonality).

To derive a finite-element approximation of this formulation, we have now to choose discrete fields and test-functions, which verify a uniform, discrete inf-sup condition. The Taylor-Hood, $P_{2}$-iso- $P_{1}$ Finite Element retains our attention here, first because it fulfill this condition (cf. [8]). Moreover, it allows to build diagonal mass matrices, when suitable quadrature formulas are used (cf. [5]). Thus, the solution to the linear system, which involves the mass matrix, is straightforward. Since there is one singularity, we can write $\mathcal{B}_{S}(t)=\kappa(t) \mathbf{y}_{S}$, where $\left(\mathbf{y}_{S}\right)$ denotes the basis of the discrete singular space, and $\kappa$ is a continous time-dependent function. Next, discretizing in time this formulation with the help of the wellknown leap-frog scheme, this results in the following fully discretized scheme:

$$
\begin{aligned}
\mathbb{M}_{\Omega} \boldsymbol{B}_{R}^{n+1}+\mathbb{M}_{R S} \boldsymbol{\kappa}^{n+1}+\mathbb{L}_{\Omega} \boldsymbol{p}^{n+1} & =\boldsymbol{F}^{n}, \\
\mathbb{M}_{R S}^{T} \boldsymbol{B}_{R}^{n+1}+\mathbb{M}_{S} \boldsymbol{\kappa}^{n+1}+\mathbb{L}_{S} \boldsymbol{p}^{n+1} & =\boldsymbol{G}^{n}, \\
\mathbb{L}_{\Omega}^{T} \boldsymbol{B}_{R}^{n+1}+\mathbb{L}_{S}^{T} \boldsymbol{\kappa}^{n+1} & =0 .
\end{aligned}
$$

Above $\mathbb{M}_{\Omega}$ denotes the usual mass matrix, and $\mathbb{L}_{\Omega}$ corresponds to the divergence term involving $\mathbf{y}_{R}^{h}$ and $p_{h}(t)$. Then, $\mathbb{M}_{R S}$ is a rectangular matrix, which is obtained by taking $\mathbf{L}^{2}$ scalar products between regular and singular basis functions, $\mathbb{M}_{S}$ is the "singular" mass matrix, and finally, $\mathbb{L}_{S}$ corresponds to the divergence term involving $\mathbf{y}_{S}^{i}$ and $p_{h}(t)$.

One can solve this system by removing the unknown $\boldsymbol{\kappa}^{n+1}$. To that aim, replace equation (12) by (12) $-\mathbb{M}_{R S} \mathbb{M}_{S}^{-1}(\underline{13})$, and equation (14) by (14) $-\mathbb{L}_{S}^{T} \mathbb{M}_{S}^{-1}(13)$. 
In this modified system, only the unknowns $\left(\boldsymbol{B}_{R}^{n+1}, \boldsymbol{p}^{n+1}\right)$ appear. If one lets $\sim$ stand for the modified matrices and right-hand sides, it reads

$$
\begin{aligned}
& \widetilde{\mathbb{M}} \boldsymbol{B}_{R}^{n+1}+\widetilde{\mathbb{L}} \boldsymbol{p}^{n+1}=\widetilde{\boldsymbol{F}^{n}}, \\
& \widetilde{\mathbb{L}}^{T} \boldsymbol{B}_{R}^{n+1}-\mathbb{L}_{S}^{T} \mathbb{M}_{S}^{-1} \mathbb{L}_{S} \boldsymbol{p}^{n+1}=\widetilde{\boldsymbol{H}^{n}}
\end{aligned}
$$

Its solution can be obtained with the help of a Uzawa-type algorithm. Finally, one concludes the time-stepping scheme by computing $\boldsymbol{\kappa}^{n+1}$ with the help of (13).

\section{Numerical Experiments}

We study now the influence of the frequency of the incoming signal, on the localization in space of the singular effects. This is of importance, since not taking into account the singular part of the electromagnetic field can result in a computed solution, which is wrong over the whole domain.

We consider an L-shaped domain $\Omega$, with a boundary $\Gamma$ split into two parts, $\Gamma=\Gamma_{C} \cup \Gamma_{A}$. On $\Gamma_{C}$ (top and bottom parts), a perfect conducting boundary condition is imposed. An incident wave enters the waveguide through the boundary $\Gamma_{A}^{i}$ (left side), and exists through $\Gamma_{A}^{a}$ (rigth side). This behavior is modelled thanks to the boundary condition (11) with a right hand-side equal to $C \sin (\omega t)$ on $\Gamma_{A}^{i}$ and zero on $\Gamma_{A}^{a}$. Initial conditions are uniformly set to zero. Above, $C$ is a constant, and $\omega$ is associated to a frequency $\nu$, which can vary.

We compare two numerical solutions. One, which is obtained by taking into account the singular part, i.e. with the Singular Complement Method or SCM. The other one, by computing only a $P^{1}$, Lagrange finite element approximation. The values of $\nu$ are set successively to $\nu_{1}=5.10^{9} \mathrm{~Hz}$, and $\nu_{2}=15.10^{9} \mathrm{~Hz}$. In each case, the mesh is such that the number of discretization nodes per wavelength is constant. Numerical dispersion, if it occurs, is therefore comparable.

Results are shown on Figure 1, For the higher frequency $\nu_{2}$, the height is roughly equal to seven wavelengths and the singular behavior is more localized, near the reentrant corner. Results are rather close. But for $\nu_{1}$, which corresponds
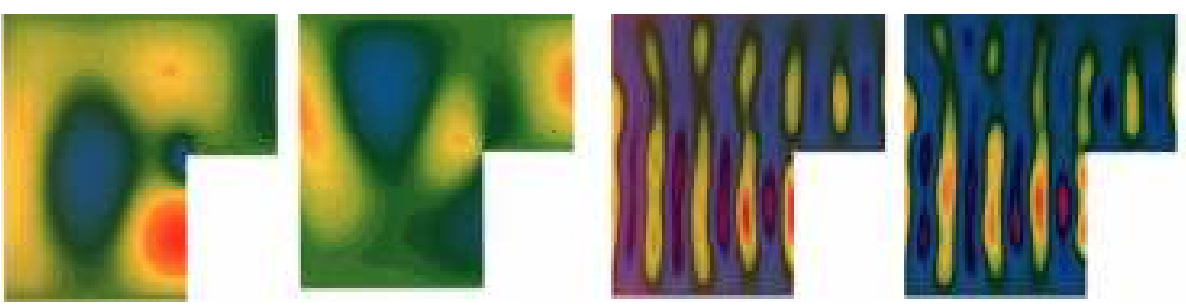

Fig. 1. with (left) and without (right) $S C M$ for low frequency $\nu_{1}$ - with (left) and without (right) $S C M$ for high frequency $\nu_{2}$ 
to a wavelength comparable to the dimensions of the domain (the height is roughly equal to two wavelengths), the solutions are very different.

\section{Conclusion}

In this paper, we were interested in the propagation of a wave in a singular waveguide, by studying how the frequency of the ingoing electromagnetic waves influences the singular solution. We developed a numerical method, based on direct, and possibly orthogonal, splittings of the space of electromagnetic solutions. One of the foremost result is that the singular behavior of the solution is more localized, near the reentrant corner for the higher frequencies than for the lower.

\section{References}

1. F. Assous, P. Ciarlet, Jr., Solving Vlasov-Maxwell equations in singular geometries, accepted to Math. and Comput. in Simulation.

2. F. Assous, P. Ciarlet, Jr., E. Garcia, A characterization of the singular electromagnetic fields by an inductive approach, submitted to Math. Meth. Appl. Sci..

3. F. Assous, P. Ciarlet, Jr., E. Garcia, J. Segré, Time dependent Maxwell's equations with charges in singular geometries, Comput. Methods Appl. Mech. Engrg., 196, 665-681, 2006.

4. F. Assous, P. Ciarlet, Jr., J. Segré, Numerical solution to the time-dependent Maxwell equations in two-dimensional singular domain: The Singular Complement Method, J. Comput. Phys., 161, 218-249 (2000).

5. F. Assous, P. Degond, E. Heintzé, P. A. Raviart, J. Segré, On a finite element method for solving the three-dimensional Maxwell equations, J. Comput. Phys., 109, 222-237 (1993).

6. I. Babuska (1973), The finite element method with Lagrange multipliers, Numer. Math., 20, 179-192 (1973).

7. F. Brezzi, On the existence, uniqueness and approximation of saddle point problems arising from Lagrange multipliers, RAIRO Anal. Numér., 129-151, (1974).

8. P. Ciarlet, Jr., V. Girault, Inf-sup condition for the 3D, $P_{2}$-iso- $P_{1}$, Taylor-Hood finite element; application to Maxwell equations, C. R. Acad. Sci. Paris, Ser. I, 335, 827-832 (2002).

9. E. Garcia, Résolution des équations de Maxwell avec charges dans des domaines non convexes, PhD Thesis, University Paris 6, France (2002). (in French)

10. P. Grisvard, Singularities in boundary value problems, 22, RMA Masson, Paris (1992). 